\title{
Computational Model for Aircraft's Takeoffs Pattern Recognition
}

\author{
Arturo Rojo Ruiz, Luis P. Sánchez Fernandez, Edgardo Felipe-Riverón, \\ and Sergio Suárez Guerra
}

Center for Computing Research, National Polytechnic Institute, Mexico

Av. Juan de Dios Bátiz s/n casi esq. Miguel Othón de Mendizábal, Col. Nueva Industrial Valle-

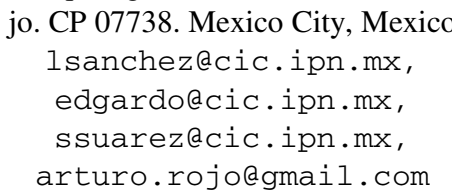

\begin{abstract}
This paper presents a novel computational multimodal model designed for pattern recognition of aircrafts' noise in real environments; with an $88.5 \%$ of effectiveness, it considers 13 different categories of aircrafts. This method includes measurements of signals of the noise produced during the takeoff at 25,000 samples per second and with a resolution of 24 bits, an spectral analysis made by means of an autoregressive model, an octave analysis, a normalization method created specifically for this work and two feed-forward neural networks. All the signals used for the design and evaluation of the results were obtained by means of field measurements.
\end{abstract}

Keywords: aircraft, noise, pattern, recognition, monitoring.

\section{Introduction}

Aircraft identification by means of the use of the spectrum characteristics of the aircraft-generated noise becomes a complicated problem when it is done in real environments because the background noise, the weather, the speed of the takeoff and even the aircraft's load can interfere with the correct detection.

Recently in the market, some measurement equipments that use neural networks to identify the class of noise have appeared. However, they can only distinguish between jet aircrafts, propeller aircrafts, helicopters and background noise [1].

A computational model is a computer program that attempts to simulate an abstract model of a particular system [2]. In this paper, the objective of the abstract model is to achieve the correct aircraft identification in real environments using only the input noise. This abstract model consists of a multimodal model that combines different signal techniques to improve the identification level, such as octave analysis and the autoregressive model. The classification suggested in this paper is divided into 13 categories.

The aircraft-generated noise samples used for this research were taken at $25 \mathrm{KHz}$ during 24 seconds in the International Airport of Mexico City during the takeoffs. 


\section{Aircraft-Generated Noise Characteristics}

The takeoff noise is considered a non stationary transient signal because it starts and ends in a zero level and it has a finite duration.

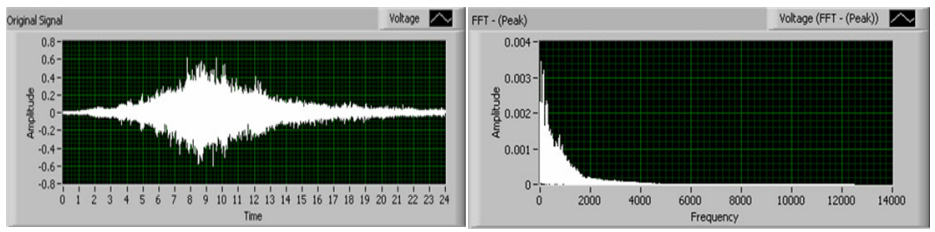

Fig. 1. Signal and spectrum of frequencies of the takeoff noise of a MD87

As Fig. 1 shows, most of the energy of the signal is below $2 \mathrm{KHz}$. In this case, apart from the fact that the signal starts and ends in a zero level, the background noise is more notorious in the ends of the signal because in the central part, the aircraft-generated noise masks it. This is why this signal is described by the following Eq. (1).

$$
S(t)=\alpha_{0} \mathrm{~N}_{\text {aircraft }}(\mathrm{t})+\alpha_{1} \mathrm{~N}_{\text {source } 1}(\mathrm{t})+\cdots+\alpha_{\mathrm{n}} \mathrm{N}_{\text {source } n}(\mathrm{t})
$$

The addition of the different noise sources, which are modified by an $\mathbf{a}$ factor that depends, among others, on the weather and the Doppler Effect.

\section{Signal's Features Extraction}

\subsection{Pre-processing}

- Application of a low-pass filter with a $2 \mathrm{Khz}$ cut-off frequency. This filter avoids the effect of high frequencies during the identification because the noise measurements are done at, at least, $200 \mathrm{~m}$ off the source. This makes uncertain the fact that the high frequencies obtained during measurement are really from the aircraft because those are the more attenuated depending on the distance.

- Frequency weighting filter C. This filter attenuates low frequencies, helping to keep stable the form of the spectrum before different samples of the aircraft because the response of the microphone to low frequencies is not always adequate, especially under $20 \mathrm{~Hz}$. This filter was created to model the response of the human ear to sounds of great intensity. It is used to evaluate environmental sounds and low frequency sounds in the audible frequencies band [3]. The calculation of the frequency weighting filter $C$ values is based on Eq. (2) [4]:

$$
C(f)=20 \log \frac{12286.62 f^{2}}{\left(f^{2}+20.6^{2}\right)\left(f^{2}+12200^{2}\right)}
$$


- Centering of the signal. Look for the point with the highest intensity and see which of the ends is the nearest, taking the same number of points towards the opposite side. This is helpful for two reasons. First, because it helps to eliminate the noise that is more notorious in the ends because in the centre the aircraft-generated noise masks the background noise. Second, it prepares the signal so that a window can be applied to it. After this, the signal is normalized.

- Separation of the background noise. In order to separate the background noise, the standard deviation of the signal has to be calculated along with the values that are less to the standard deviation; the highest values are taken to zero. This is done under the premise that the information held in the values less to the standard deviation is background noise. The standard deviation is calculated by Eq. (3).

$$
\sigma=\sqrt{\frac{1}{N} \sum_{i=1}^{N}(x i-\bar{x})^{2}}
$$

- Application of a Gauss window. Apply a Gauss window to the two signals that exist: the signal of the aircraft-generated noise and the resulting signal after the separation from the background noise. This stands out the spectrum characteristics in the center of the signal, where the highest energy of the signal is. The calculation of the Gauss window is based on Eq. (4).

$$
W_{g}(x)=e^{\frac{-\left(x^{2}\right)}{2 \sigma^{2}}}
$$

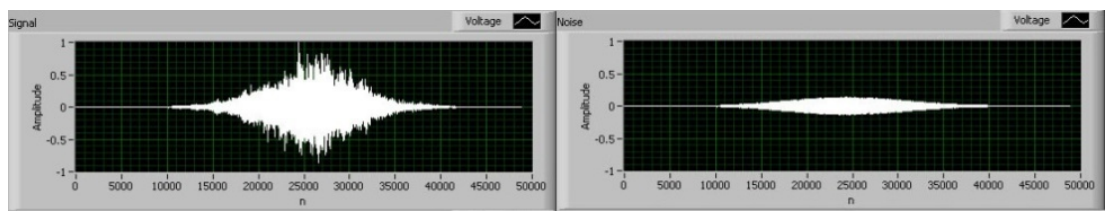

Fig. 2. Pre-processed signal and background noise

\subsection{Processing}

\subsubsection{Autoregressive Model}

The autoregressive model is used for the pre-processed signal in order to model the aircraft-generated noise behavior. This model is used by the LPC (Linear Prediction Code) to describe the vocal tract as an IIR (Impulse Infinite Response) filter and it considers that a sound can be described as an IIR filter with a white noise entrance, which, when passing through it, generates sound [5], [6]. This filter is described by Eq. (5).

$$
H_{S}(z)=\frac{1}{\sum_{i=0}^{M} a_{i} z^{-i}}=\frac{1}{A(z)}
$$


Therefore, the first thing to do is to calculate $A(z)$, which are the filter coefficients. $\mathrm{A}(\mathrm{z})$ vector is calculated by solving the Eq. (6) using the Levinson - Durbin [6] method.

$$
\left[\begin{array}{cccc}
R s s(0) & R s s(-1) & \ldots & R s s(-(p-1)) \\
R s s(1) & R s s(0) & \ldots & R s s(-(p-2)) \\
\vdots & \vdots & \ldots & \vdots \\
R s s(p-1) & R s s(p-1) & \ldots & R s s(0)
\end{array}\right]\left[\begin{array}{c}
a_{1} \\
a_{2} \\
\vdots \\
a_{p}
\end{array}\right]=-\left[\begin{array}{c}
R s s(1) \\
R s s(2) \\
\vdots \\
R s s(p)
\end{array}\right]
$$

$\operatorname{Rss}(\mathrm{k})=\operatorname{Rss}(-\mathrm{k})$ therefore $\operatorname{Rss}(-1)=\operatorname{Rss}(1)$ and so on

To get the Rss(k) values, Eq. (7) is used. The $1 / \mathrm{N}$ factor is the factor of the partial autocorrelation scale, which guarantees the coefficients stability [6].

$$
\operatorname{Rss}(\mathrm{k})=\frac{1}{\mathrm{~N}} \sum_{\mathrm{i}=0}^{\mathrm{N}-1-|\mathrm{k}|} \mathrm{S}(\mathrm{i}+|\mathrm{k}|) \mathrm{S}(\mathrm{i}) \quad \text { for }|\mathrm{k}|<\mathrm{p}+1
$$

A "p" number has to be chosen. The bigger this value is, the filter's frequency response will be more approximated to the FFT. In this case, the number used was $p=$ 310 (it was obtained by means of experimentation). It was chosen because if $\mathrm{p}$ were a lower value, some characteristics would be lost, and if it were a higher value, it would be more susceptible to the variations between patterns of the same class, which can be produced by the wind, humidity, the speed of the takeoff, or just the differences existent between engines. At the same time, it lets us differentiate between the diverse classes of aircrafts.

The obtention of the frequency response $\mathrm{H}(\mathrm{w})$ of the IIR filter is based on Eq. (8). The coefficients obtained after applying the FFT are divided term by term.

$$
H(w)=\frac{F F T(1)}{F F T(A(z))}
$$

A FFT of 4096 was used because it allows good precision with few values.

Now, the magnitude of each term of the vector given by Eq. (9) has to be calculated.

$$
|H(w)|=\sqrt{R e^{2}+I m^{2}}
$$

When the FFT of 4096 is applied to a signal sampled at $25,000 \mathrm{~Hz}$, the result is 2048 coefficients that are equivalent to $12,500 \mathrm{HZ}$. A great number of the 2048 values are zeros and the other values do not allow us to distinguish between classes; this is why only the first 140 points were taken, because there is where the biggest differences between classes can be found.

After this, the autoregressive model is applied to the two signals obtained from the pre-process that are shown in Fig. 2.

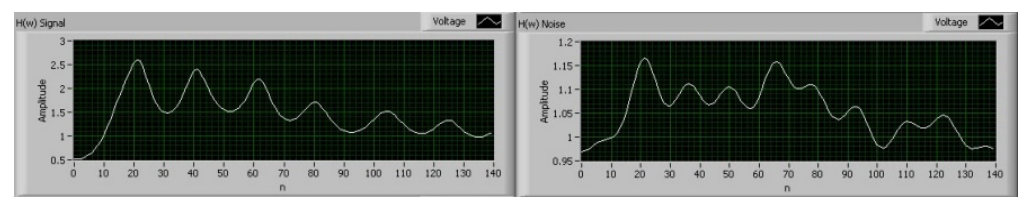

Fig. 3. Frequency response of the signal and the background noise 
Now, the $\mathrm{H}(\mathrm{w})$ coefficients of the background noise have to be subtracted from the $\mathrm{H}(\mathrm{w})$ coefficients of the signal.

Due to the subtraction some of the coefficients might be negative, in which case, they are taken as a 0 . This subtraction attenuates the frequencies introduced by the background noise in the aircraft-generated noise.

Finally, a normalization method created specifically for this work .The $\mathrm{H}(\mathrm{w})$ has to be normalized by means of the following:

- All the points of the $\mathrm{H}(\mathrm{w})$ are divided by the maximum value of $\mathrm{H}(\mathrm{w})$, as shown in Eq. (10).

$$
H_{\text {norm }}(w)=\frac{H(w)}{\operatorname{MAX}(H(w))}
$$

- The mean and the Standard deviation of Hnorm(w) are calculated; after that, Eq. (11) is applied.

$$
H_{\text {norm } 2}(w)=\frac{H_{\text {norm }}(w)}{(\mu+\sigma) x \alpha}
$$

This, considering $\mu$ to be the mean, $\sigma$ the Standard deviation of $H_{\text {norm } 2}$ and $\alpha=12$ is a factor to make that most of the $\mathrm{H}_{\text {norm } 2}(\mathrm{w})$ values are less than 1 .

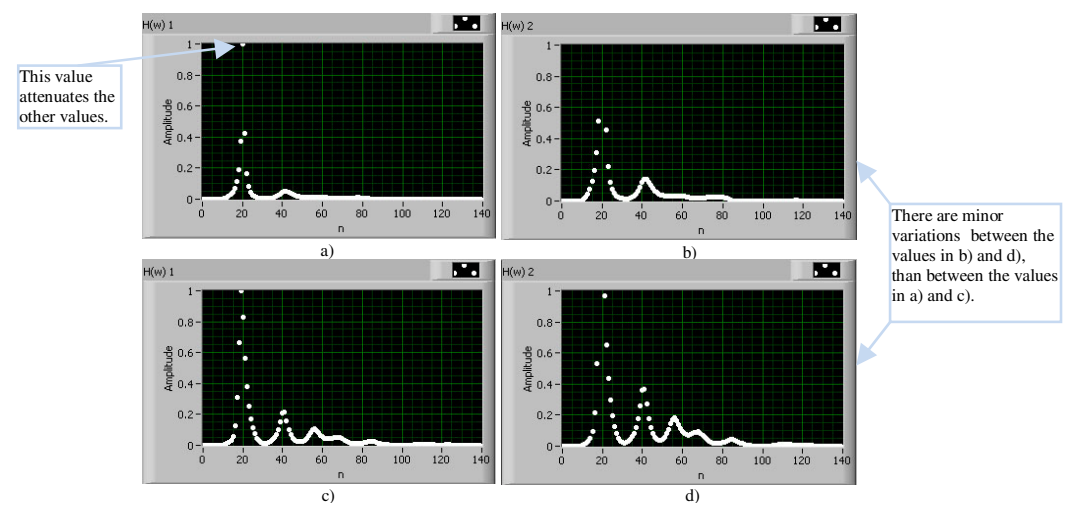

Fig. 4. a) ATR-42 (1) with classic normalization, b) ATR-42 (1) with the proposed normalization, c) ATR-42 (2) with classic normalization, d) ATR-42 (2) with the proposed normalization

Fig. 4 shows two aircrafts ATR-42, with big variations between them. It can be observed that in the classic normalization a) and c) (only Ec. 10), the second peak is multiplied by 4 ; it changes from 0.05 to a little over 0.2 . On the other hand, if the proposed normalizations b) and d) are used, the variation is from 0.15 to a little over 0.35 , so, it is multiplied by a factor of 2.33 . The differences between the two ATR-42 are caused only by component $n=20$. As shown in a), this component is very big; thus, it attenuates the other points. However, when using the proposed normalization, although component $n=20$ is out of range, it has a value of 1.78 , the other points are less affected, so, they remain stable and recognition is made easier. 


\subsubsection{Octave Analysis}

To complete the information obtained by using the autoregressive model, the $1 / 12$ octave analysis is used in order to get patterns and train a second neural network because although the auto-regressive model provides lineal frequency information, there is information that can go unnoticed. This is not the case of the $1 / 12$ octave analysis because a logarithmic scale is used. The 1/12 analysis was used because other analysis such as $1 / 3$ or $1 / 6$ get few values and do not permit the classification and if the $1 / 24$ is used the bands are too narrow.

For this analysis, the bands of frequencies $(\mathrm{Hz})$ were used centered in: 129,136 , $144,153,162,172,182,193,204,216,229,243,257,273,289,306,324,343,364$, 386, 408, 433, 459, 486, 510, 550, 580, 610, 650, 690, 730, 770, 820, 870, 920, 970, $1030,1090,1160,1220$. The calculation of all this is based on references [7], [8].

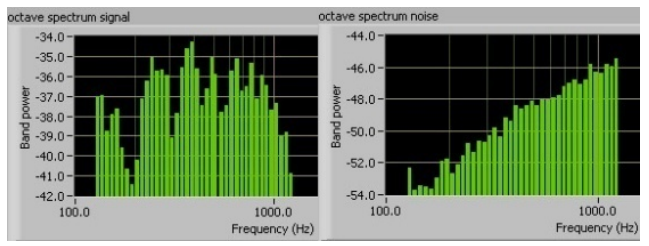

Fig. 5. Resulting values of the $1 / 12$ Octave Analysis. Left: values of the aircraft's noise signal, right: values of the background noise.

The resulting values are not practical to use in a neural network, because they are negative and with very dispersed values. Therefore, they have to be conditioned in the following way:

- Apply Eq. (12).

$$
O\left(f_{i}\right)=\left|\frac{1}{\text { Band Power }\left(f_{i}\right)}\right|
$$

Thus, it makes the values positive while keeping the same form.

- $\quad \mathrm{O}(\mathrm{f})$ coefficients of the background noise are subtracted from the $\mathrm{O}(\mathrm{f})$ coefficients of the signal.

- $\quad$ Normalization using Eq. (9) y Eq. (10) but with $\alpha=3$.

This results in 40 values which are used to train a neural network.

\section{Results}

Using this methodology there were created two Backpropagation NNs (Neural Network). The first, using the obtained values of the auto-regressive model, has 140 input neurons, 27 middle neurons and 13 output neurons. The second network counts with 40 input neurons, 27 middle neurons and 13 output neurons. There were used 55 training patterns, 6 per class, which cause repeated patterns in certain classes. 


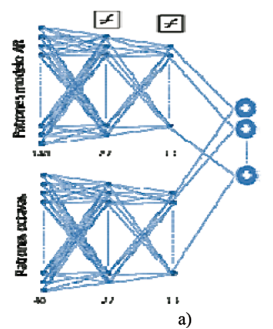

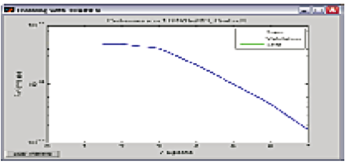

b)

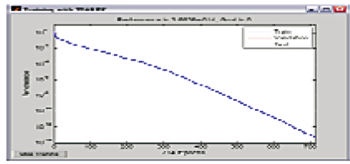

c)

Fig. 6. a) Neural model topology for aircraft recognition, b) Results of the training of the NN $140 \times 27 \times 13, c)$ Results of the training of the NN 40x27x13

An $88.5 \%$ of effectiveness was achieved when proving the model with 35 test patterns. It is important to remark that these patterns are contaminated with voices, barks, bird's sounds, background music, etc. and they were obtained under different weather conditions during 4 different measurements (June $25^{\text {th }}$, 2007; July $6^{\text {th }}, 2007$ and December $13^{\text {th }}$ and $\left.21^{\text {st }}, 2007\right)$.

Table 1. Mistakes per category

\begin{tabular}{|l|c|c|c|c|}
\hline Category & $\begin{array}{l}\text { Total No. of } \\
\text { patterns. } \\
\text { (Training } \\
\text { patterns) }\end{array}$ & $\begin{array}{l}\text { No. of errors } \\
\text { using the AR } \\
\text { (Autoregressive } \\
\text { Model) }\end{array}$ & $\begin{array}{l}\text { No. of } \\
\text { errors using } \\
\text { Octave } \\
\text { Analysis. }\end{array}$ & $\begin{array}{l}\text { No. of errors using the } \\
\text { addition of the outputs of the } \\
\text { Neural Networks of the AR } \\
\text { Model and Octave Analysis. }\end{array}$ \\
\hline Airbus 1 & $5(4)$ & 0 & 1 & 0 \\
\hline Airbus 2 & $4(3)$ & 0 & 0 & 0 \\
\hline Airbus 3 & $13(6)$ & 1 & 4 & 3 \\
\hline Airbus, Boeing 737-800 & $10(6)$ & 2 & 1 & 0 \\
\hline Atr-42 & $2(2)$ & 0 & 0 & 0 \\
\hline Boeing 737-100, 737-200 & $2(2)$ & 0 & 0 & 0 \\
\hline Boeing 737-600, 737-700 & $13(6)$ & 0 & 1 & 0 \\
\hline Boeing 747-400 & $1(1)$ & 0 & 0 & 0 \\
\hline Fokker F100 & $8(5)$ & 0 & 1 & 0 \\
\hline Fokker F100 2 & $2(2)$ & 0 & 0 & 0 \\
\hline Fokker F100, Boeing 737- 200 & $9(6)$ & 0 & 1 & 0 \\
\hline Fokker F100, Boeing 737-2002 & $11(6)$ & 1 & 1 & 1 \\
\hline MD87, MD88 & $10(6)$ & 1 & 3 & 4 \\
\hline & & & & 0 \\
\hline Total & $90(55)$ & 5 & 13 & 0 \\
\hline
\end{tabular}

As table 4.1 shows the errors are different; that is, each technique allows a better identification of certain classes. The simple addition of the outputs of the two neural networks permits the obtention of a yield superior to the one obtained by using the techniques separately.

Fig. 7 shows one of the sample patterns used, which, lacking a considerable part of the signal, was correctly classified as a MD87.

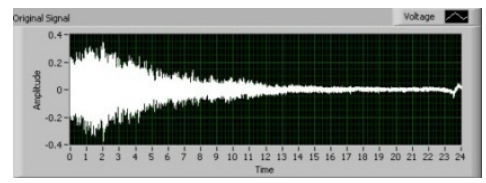

Fig. 7. Example of a sample noise produced by a MD87 


\section{Conclusions and Future Work}

In spite of having complicated circumstances due to the background noise present in the noise measurements, which can be generated by a great number of different sources, with the proposed computational model good results can be obtained (even better than when using the method shown in [9] without these circumstances interfering with the identification.

In addition, a test was made, diminishing the sample frequency to $12500 \mathrm{~Hz}$, the AR model with $\mathrm{p}=155$ and a FFT of 2048, in order to keep the same $\Delta \mathrm{f}$. In this case similar results were obtained, with a diminution of $75 \%$ of the processing time.

For future work, the differences of the yield when applying this technique after segmenting the original signal will be analyzed; new parameters to create the neural network should be tested. For example, the area under the curves found in the response of frequency $\mathrm{H}(\mathrm{w})$ of the IIR filter that modelates the noise. Also, a third neural network that joins the two existent networks should be created. It is also necessary to increase the number of training and test patterns.

\section{References}

1. Lochard - Airport Environment Management: EMU2100 Brochure (2008)

2. Humphreys, P.: Extending Ourselves: Computational Science, Empiricism, and Scientific Method. Oxford University Press, Oxford (2004)

3. Holding, J.M.: Aircraft noise monitoring: principles and practice. IMC measurement and Control 34(3), 72-76 (2001)

4. International Electrotech. Comm (IEC): Standard IEC651: Sound Level Meters (1979)

5. Chu, W.: Speech Coding Algorithm: Foundation and Evolution of Standardized Coders. J. Wiley, Chichester (2003)

6. Perez-Meana, H. (ed.): Advances in Audio and Speech Signal Processing: Technologies and Applications. Idea Group Pub. (2007)

7. International Electrotech. Comm (IEC): Standard IEC1260: Octave Filters (1995)

8. American National Standards Institute (ANSI): Standard S1.11-2004: Specification for Octave-Band and Fractional-Octave-Band Analog and Digital Filters (2004)

9. Sanchez, L., et al.: Noise pattern recognition of airplanes taking off: task for a monitoring system. In: Rueda, L., Mery, D., Kittler, J. (eds.) CIARP 2007. LNCS, vol. 4756, pp. 831840. Springer, Heidelberg (2007) 